\title{
Productivités horaires du trayeur dans deux installations de traite de petites dimensions pour brebis laitières
}

\author{
J. LE DU \\ avec la collaboration technique de J.F. COMBAUD, P. LAMBION, Y. DANO, \\ G. GUIONNEAU et P. ROLLAND \\ INRA, Laboratoire de Recherches sur la Traite \\ 65, rue de Saint-Brieuc, F 35042 Rennes Cedex
}

\begin{abstract}
Résumé
La productivité horaire du trayeur est étudiée dans 2 petites installations de traite pour brebis :

- Installation $12 \times 24$ : fosse de type "Casse 》 constituée de 2 quais équipés chacun de 12 stalles et de 6 faisceaux trayeurs soit au total 12 faisceaux pour 24 stalles.

- Installation $12 \times 12$ : un seul des 2 quais équipés de 12 stalles et de 12 faisceaux trayeurs est utilisé.

Cette comparaison montre que :

- La productivité est plus élevée $(\mathrm{P}<0,001)$ en $12 \times 24$ qu'en $12 \times 12 \quad(182$ contre 157 brebis/heure). Les temps de sortie des brebis sont plus courts et le trayeur travaille plus rapidcment en ayant un faisceau trayeur pour 2 stalles $(12 \times 24)$.

- Avec des intervalles de 9 et 15 heures entre traites, la productivité est plus élevée le soir que le matin $(177$ contre 162 brebis/heure) $(P<0,001)$. Le matin avec des mamelles plus congestionnées, l'égouttage est plus difficile à effectuer et les chutes de faisceaux sont plus fréquentes $(+43 \mathrm{p} .100)$.

- La différence entre trayeurs $(7,4$ brebis/heure) est liée à la rapidité des opérations d'admission $(P<0,05)$ et de sortie $(P<0,001)$ des brebis plus qu'à la traite (N.S.). Il importe que tous les trayeurs puissent manæeuvrer les portes, les cornadis mobiles et les distributeurs d'aliments facilement et sans quitter la fosse.

- Avcc une méthode de traite simple, de nombreuses races de brebis pourraient être traites dans des installations moins onéreuses que celles qui ont été conçues pour la brebis Lacaune. L'installation $12 \times 12$ nous semble suffisante pour certains des nombreux troupeaux de moins de 150 brebis qui restent à équiper dans le Bassin Méditerranéen.
\end{abstract}

Mots clés : Brebis, traite, salle de traite, organisation du travail, productivité.

\section{Introduction}

Les installations de traite en fosse de type «Casse» ont été développées vers 1961 pour satisfaire les exigences physiologiques spécifiques à la brebis Lacaune (Bosc, 1963). Cette race est difficile à traire à la main : 20 brebis/heure (ANON, 1967 ; 
Delmas, 1984). A la machine, elle nécessite des techniques de traite plus compliquées que toutes les autres races (BosC, 1963 ; ANON, 1967). A Roquefort, ce contexte justifie l'utilisation d'infrastructures relativement lourdes : 24 à 48 stalles réparties sur 2 quais séparés par une fosse, 12 à 24 faisceaux-trayeurs (Bosc, 1974; LE Du, 1979, 1981). Or, c'est ce système "Casse" peu modifié dans son principe qui est à l'origine de la diffusion de la traite mécanique dans les pays du Bassin Méditerranéen. En France, il représente 97 p. 100 des salles de traite de moins de 20 ans (GAUTIER, 1980).

Actuellement, il semble judicieux de faire évoluer une telle conception des chantiers de traite (Delmas \& Poussou, 1984; Le Du, 1984; Vallerand, 1984). D'une part, les techniques de traite complexes préconisées à l'origine pour la brebis Lacaune n'ont jamais été utilisées pour d'autres races. D'autre part, même à Roquefort, le progrès génétique, le perfectionnement du matériel et le souhait légitime de «traire vite» conduisent les éleveurs à simplifier leurs techniques de traite (Bosc, 1974 ; Cottier \& Delmas, 1981 ; Delmas, 1984).

Dans certaines régions de France (Pyrénées, Corse) et d'Italie (Sardaigne) puis en Espagne et en Grèce, Ja demande potentielle en matériel de traite intéresse principalement les petits troupeaux (100 à 150 brebis) constitués de races faciles à traire (plus de 60 brebis/heure à la main). Dans ce cas, avec une installation «compacte» où 12 brebis sont disposées sur un seul quai, nous avons montré qu'un trayeur peut traire au rythme de 140 brebis/heure si la technique de traite est suffisamment simple (LE DU, 1984). A Roquefcrt, dans des condicions d'exploitation, certes plus contraignantes, cette productivité serait satisfaisante. Elle est comparable à celles que l'on observe dans les installations «Casse» plus onéreuses (Bosc, 1963 ; LE DU, 1979 ; Cottier \& Delmas, 1981).

Nous plaçant dans un contexte différent de celui de Roquefort, notre étude a pour objet de préciser l'intérêt d'une installation simplement constituée de 12 stalles et de 12 faisceaux disposés sur un seul quai en la comparant à un système "Casse » traditionnel comportant 24 stalles et 12 faisceaux répartis sur 2 quais.

\section{Matériel et méthodes}

\section{A. Installations de traite}

Une fosse de type «Casse » de 4,5 $\mathrm{m}$ de long est aménagée pour traire au choix dans les conditions suivantes (fig. 1) :

- Installation $12 \times 24$ : système «Casse» traditionnel constitué de 2 quais équipés chacun de 12 stalles et de 6 faisceaux trayeurs (24 stalles et 12 faisceaux au total).

- Installation $12 \times 12$ : un seul quai, équipé de 12 stalles et de 12 faisceaux trayeurs est utilisé.

Dans les deux cas, les quais sont équipés de cornadis mobiles permettant la contention collective des animaux et la distribution d'aliments concentrés. Le lactoduc est disposé en «ligne basse ». 


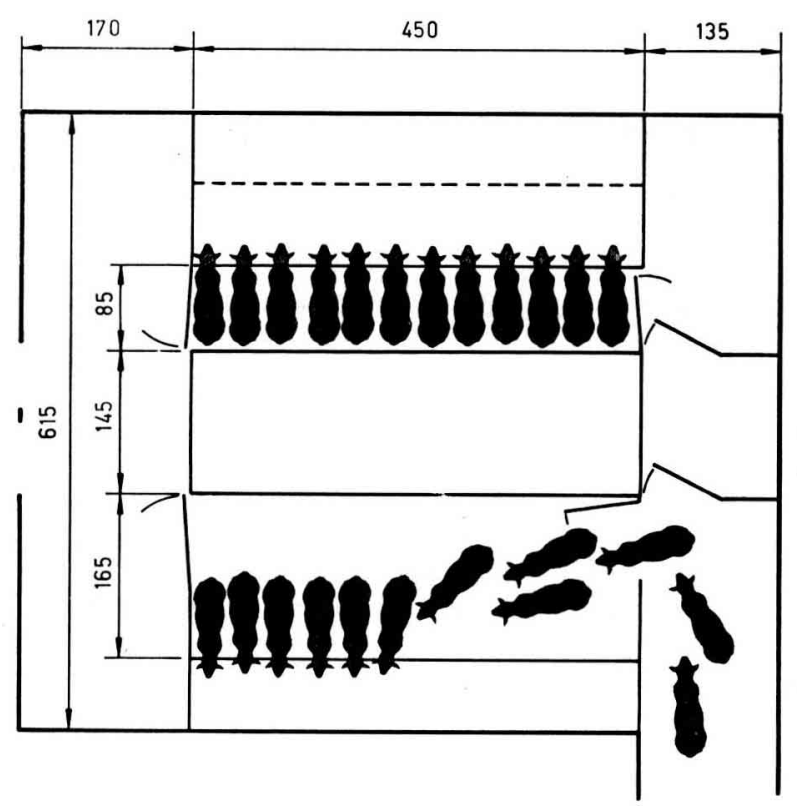

FIG. 1

Plan de l'installation de traite $12 \times 24$ (12 faisceaux-trayeurs, 24 stalles $)$ avec les dimensions en centimètres.

Quai du haut : brebis en position de traite; quai du bas : admission des brebis.

Pour l'installation $12 \times 12$,

seulement le quai du haut équipé de 12 faisceaux-trayeurs est utilisé.

Plan of the $12 \times 24$ installation (12 teatcup clusters, 24 milking stands) with dimensions in centimeters.

Upper platform : ewes in the milking position; lower platform : admission of the ewes.

For the $12 \times 12$ installation, only the upper platform with 12 units was used.

\section{B. Techniques de traite}

La traite effectuée par un seul trayeur a lieu à $7 \mathrm{~h} 30$ et $16 \mathrm{~h} 30$. Le trayeur actionne le distributeur d'aliment concentré puis 24 brebis (installation $12 \times 24$ ) ou 12 brebis (installation $12 \times 12$ ) sont admises en même temps dans la salle de traite. Lorsque les animaux sont en place, le trayeur commande la fermeture du cornadis et le met en position de traite. Le distributeur de concentré, les barrières d'admission des animaux, le cornadis mobile peuvent être manœuvrés sans quitter la fosse.

Les gobelets sont posés sans préparation du pis. Lorsque le lait cesse de couler, un égouttage à la machine est pratiqué. Le faisceau-trayeur est ensuite déposé (installation $12 \times 12$ ) ou éventuellement transféré à la brebis voisine (cas de l'installation $12 \times 24$ ). Après dépose du dernier faisceau, le - ou les - cornadis sont manæuvrés et les animaux quittent la salle de traite. 


\section{Protocole expérimental}

Les agnelages étant groupés pendant une période de 24 jours, 48 brebis de race Lacaune sont traites à la machine après 2 jours d'allaitement $\left(21\right.$ en $1^{\text {re }}$ lactation, 6 en $2^{\circ}, 4$ en $3^{\circ}, 9$ en $4^{\circ}, 7$ en $5^{\circ}$ et 1 en $6^{\circ}$ ). La période expérimentale d'une durée de 28 jours, débute 35 jours après la date moyenne des agnelages. La production laitière moyenne décroît de $989 \mathrm{ml} /$ jour à $741 \mathrm{ml} /$ jour entre le début et la fin de l'expérimentation. Du fait des horaires de traite $(7 \mathrm{~h} 30$ et 16 h 30$), 60$ p. 100 de la production laitière journalière est obtenue le matin.

Avant le début de l'étude, les animaux sont traits dans l'installation $12 \times 24$. Ensuite, chacune des deux installations étudiées est utilisée pour la traite pendant 2 périodes d'une semaine dans l'ordre suivant : $12 \times 24,12 \times 12,12 \times 12,12 \times 24$. Deux trayeurs participent à l'expérimentation : le $1^{\text {er }}$ trayeur pendant les 2 premières semaines, le second pendant les 2 semaines suivantes. Les animaux qui ne participent pas à l'expérimentation sont traits avant le groupe expérimental et les changements d'installation ont lieu après la traite du vendredi soir. Le relevé des différents critères mesurés est effectué à chaque traite du lundi matin au vendredi soir.

\section{Temps de travail et productivité}

Un chronométreur note pour chaque série de 12 brebis (installation $12 \times 12$ ) ou 24 brebis (installation $12 \times 24$ ) :

- le temps d'entrée : intervalle entre le début de la distribution de concentré et la pose du premier faisceau-trayeur. Ce temps comprend la distribution du concentré, l'admission des animaux, leur contention collective et la manouvre du cornadis,

- le temps de travail : intervalle entre la pose du $1^{\text {er }}$ faisceau et la dépose du dernier faisceau sur une série de brebis (12 ou 24),

- le temps de sortie : intervalle entre la dépose du dernier faisceau sur une série de brebis (12 ou 24) et le début de la distribution de concentré pour la série suivante.

Pour la présentation des résultats, ces temps sont exprimés en secondes pour 24 brebis. A chaque traite, pour chaque paramètre, 2 mesures sont obtenues : elles correspondent en $12 \times 24$, aux 2 séries de 24 brebis. En $12 \times 12$, les temps des séries 1 et 2 ( $1^{\text {re }}$ mesure) puis 2 et 3 ( $2^{*}$ mesure) sont additionnés.

Ces mesures permettent de calculer la productivité horaire : nombre de brebis traites par homme et par heure. La productivité est égale à 3600 multiplié par 24 et divisé par la somme du temps de travail, du temps d'entrée et du temps de sortie exprimés en secondes.

\section{Chutes de faisceaux-trayeurs}

Pcur mesurer la fréquence des chutes de faisceaux-trayeurs, la traite d'une brebis est notée 0 si elle s'effectue sans chutes et 1 lorsqu'il s'en produit une ou plusieurs quelle qu'en soit la cause. Pour chaque série de 24 brebis, le résultat est exprimé en nombre de chutes pour 100 traites. 


\section{Méthodes d'analyse statistique}

Pour chacun des paramètres étudiés, la variance totale est décomposée pour calculer l'effet des 3 facteurs contrôlés (installation, trayeur, horaire) et les 3 interactions de $1^{\mathrm{er}}$ ordre. La variance attachée à l'interaction de $2^{\mathrm{e}}$ ordre est confondue avec celle de l'erreur résiduelle.

\section{Résultats et discussion}

A) L'installation conventionnelle à 12 postes pour 24 stalles permet de traire 182,3 brebis/heure en moyenne contre 156,9 brebis/heure pour l'installation $12 \times 12$ (tabl. 1). Deux facteurs expliquent cet accroissement significatif $(P<0,001)$ de 25,4 brebis/heure en $12 \times 24$ :

- la libération des animaux se faisant en manœuvrant successivement les cornadis «droite» et «gauche», la seconde série de 12 brebis est libérée alors que l'évacuation de la première série n'est pas terminée. Du fait de ce «chevauchement », le temps de sortie de 24 brebis est significativement plus court $(P<0,001)$ que dans l'installation $12 \times 12$ où les brebis sont manipulées par lots successifs de 12 animaux;

- avec un faisceau pour 2 stalles (simple équipement) le trayeur après avoir trait les brebis «paires» transfère celui-ci sur les brebis «impaires » sans avoir à le poser sur son support. Ce transfert, plus rapide qu'une pose et une dépose effectuées séparément diminue le temps de travail $(P<0,001)$.

L'examen de la distribution globale des productivités montre que celles-ci sont nettement moins dispersées en $12 \times 24$ (fig. 2). Finalement, toutes les traites se déroulent à un rythme supérieur à 150 brebis/heure. Par contre, en $12 \times 12$, cette productivité est dépassée dans 60 p. 100 des cas seulement. Ce constat, peut être un élément déterminant pour guider le choix de l'éleveur.

En $12 \times 12$, on note que les productivités sont respectivement supérieures de 14 et 19 brebis/heure à celles que les mêmes trayeurs avaient obtenues antérieurement dans notre élevage avec un cornadis fixe et une distribution manuelle du concentré (LE Du, 1984). Ce gain provient d'une diminution de 35 p. 100 du temps d'entrée (1). Le cornadis mobile et une certaine mécanisation de la distribution du concentré apparaissent donc essentiels pour valoriser une petite installation à un seul quai.

B) Le trayeur affecte peu $(7,4$ brebis/heure) mais significativement la productivité (tabl. 1) Avec le trayeur le plus lent, la diminution de productivité est due, à parts sensiblement égales, aux augmentations du temps de travail $(+8,5 \mathrm{~s})$, du temps d'entrée $(+7,4 \mathrm{~s})$ et du temps de sortie $(+7,0 \mathrm{~s})$. La technique de traite étant très simple (égouttage machine seulement) et appliquée de façon stricte, l'accroissement du temps de travail faible en valeur relative $(2,2$ p. 100) n'est pas significatif. Par contre, les accroissements du temps d'entrée $(+8,7$ p. 100) et de sortie $(+19,1$ p. 100) sont significatifs. Selon les intéressés, ils résulteraient des difficultés qu'éprouve le trayeur le plus petit $(1,67$ contre $1,77 \mathrm{~m})$ à manœuvrer sans quitter la fosse, les

(1) Ce temps comprend la distribution de concentré, l'admission des animaux, leur contention et la manouvre du cornadis. 
portes servant à la circulation des animaux, le distributeur de concentré et le cornadis mobile. Or, ces composants de notre installation sont identiques à ceux qui sont les plus répandus dans la région de Roquefort. Pour rentabiliser une installation de traite, certains aménagements restent à prévoir pour que le maximum de manœuvres soit effectué sans quitter la fosse, quelles que soient la morphologie, la force ou l'adresse du trayeur.

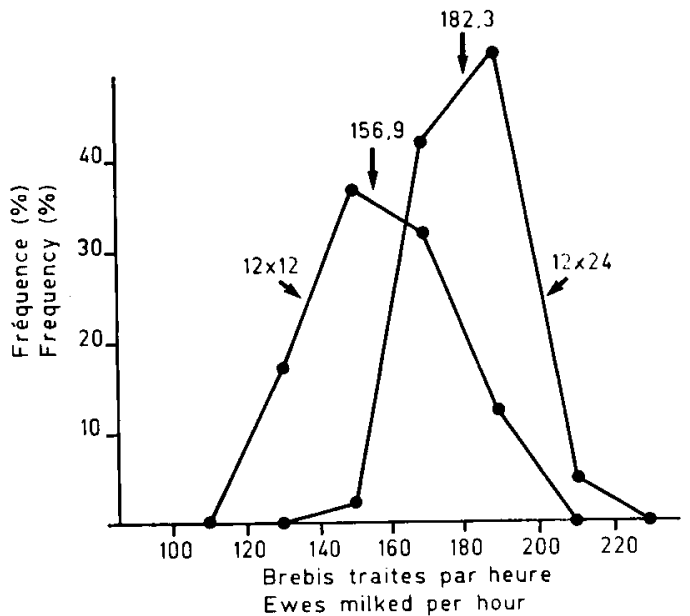

FIG. 2

Distribution des productivités horaires (brebis/heure) pour les installations $12 \times 12$ et $12 \times 24$.

Distribution of milking performance (ewes/hour) for $12 \times 12$ and $12 \times 24$ milking parlours.

C) L'horaire de traite (matin ou soir) affecte de façon significative la productivité, plus élevée de 14,7 brebis/heure, le soir où le temps de travail est diminué de 10 p. $100(\mathrm{P}<0,001)$.

L'inégalité des productions laitières du matin $(60$ p. 100) et du soir $(40$ p. 100) n'a qu'une incidence très indirecte. En effet, avec des niveaux de production (ou des temps d'émission du lait) similaires, nous avons montré que 12 faisceaux constituent un équipement suffisant pour traire sans délais d'attente entre animaux (LE Du, 1984).

Par contre, il existe 2 facteurs susceptibles d'influencer directement le temps de travail :

- les mamelles étant plus congestionnées le matin, nous avons observé que l'égouttage, plus difficile à effectuer, est légèrement prolongé ;

- nous confirmons qu'une augmentation de la consistance du pis conduit à des chutes plus fréquentes (LE Du, 1983). A la limite du seuil de signification (à $P<0,05)$, l'accroissement de 43 p. 100 de leur fréquence peut contribuer le matin à désorganiser le travail et à diminuer la cadence de traite. 


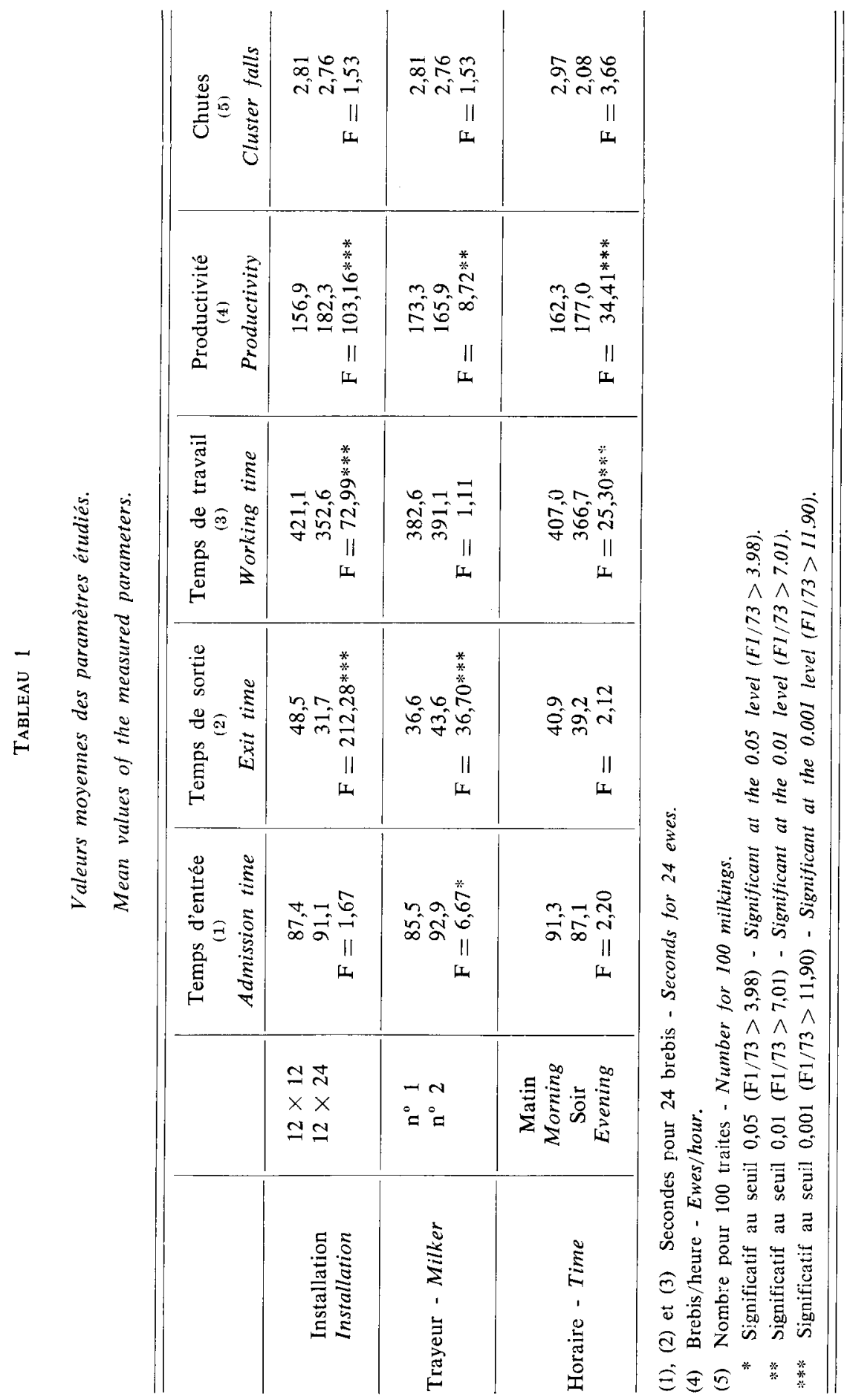


Il importe de souligner que dans nos conditions expérimentales, la fréquence moyenne des chutes de gobelets est faible (2,8 p. 100). Elle est comparable à celle que nous avons antérieurement mesurée sur notre troupeau avec le même faisceau : 1,25 p. 100 (LE Du, 1982). Dans d'autres conditions d'exploitation, en race Lacaune, des fréquences moyennes très supérieures sont couramment observées : 5 à 32 p. 100 (LE Du, 1977), 11 à 18 p. 100 (Le Du, 1983), 15 p. 100 (Labussiere, 1984). L'effet race est important : 4 à 5 p. 100 en Manech contre 11 à 18 p. 100 en Lacaune (LE Du, 1983), 0,3 p. 100 en Karagouniko, 3 p. 100 en Tsigay contre 29 p. 100 en Stara Zagora (Labussiere, 1984), 28 p. 100 avec des races d'Europe de l'Est (Mazac, Kopal \& Cerny, 1967) alors qu'avec le même matériel (2), la fréquence était de 9 p. 100 en Préalpes du Sud (LE Du et al., 1978). Une telle variabilité de la fréquence des chutes affecte inévitablement le travail du trayeur. II convient donc d'en tenir compte pour interpréter nos résultats caractérisés par des productivités horaires élevées.

\section{Conclusions}

A l'issue de ce travail, il nous semble que de nombreuses races de brebis pourraient être traites dans des installations plus simples que celles qui ont été conçues à Roquefort vers 1961 pour la brebis Lacaune. C'est notamment le cas des races Manech (Le Du, 1984), Corses (Vallerand, 1984), Sardes (CASu et al., 1978) ou plus généralement de certaines des races étudiées dans le cadre du projet M4 du réseau ovin méditerranéen de la F.A.O. (LABussiere, 1984). Toutefois, pour que la mécanisation soit rentable, il convient de tenir compte de certains constats :

A) Du fait des caractéristiques propres à chaque trayeur (taille, degré d'apprentissage, rapidité d'exécution, ...), celui-ci a une incidence sur la productivité horaire. Ainsi, la rapidité d'exécution affecte directement le temps de travail lié à la manipulation des faisceaux-trayeurs.

Mais, certaines différences de productivité proviennent aussi de la mauvaise adaptation de l'installation à la morphologie de l'individu. Un trayeur de petite taille est plus fréquemment obligé de sortir de la fosse pour manœuvrer les portillons d'accès aux quais, le cornadis mobile ou le distributeur de concentré. Une conception plus rationnelle de ces équipements accessoires est souhaitable pour que la productivité soit indépendante des caractéristiques morphologiques, de la force ou de l'adresse des trayeurs. La diminution de la pénibilité du travail serait d'ailleurs un élément favorable à l'insertion des travailleurs vieillissants ou parfois des femmes souvent exclues du travail de traite du fait de la mécanisation (CAvalie \& PASCaL, 1978).

B) Pour atteindre des productivités moyennes de l'ordre de celles que nous avons obtenues ( 157 brebis/heure en $12 \times 12$ contre 182 en $12 \times 24)$, il est essentiel de disposer d'un cornadis mobile et d'un distributeur d'aliments concentrés manæuvrables sans quitter la fosse. Ces deux équipements correspondent approximativement en $12 \times 12$ à un gain de 14 à 19 brebis/heure. II convient, par ailleurs, que la fréquence des chutes de gobelets soit faible : 2,8 pour 100 traites dans nos conditions expérimentales. Par contre, des valeurs supérieures à 10 p. 100 seraient incompatibles avec le

(2) Ancien matériel Alfa-Laval : gobelets en laiton $n^{\circ} 26895$ et manchons en caoutchouc n* 25200 . 
maintien d'une bonne organisation du travail et conduiraient à des productivités inférieures à celles que nous avons mesurées.

C) L'installation $12 \times 24$ (24 stalles et 12 faisceaux-trayeurs) est la plus rationnelle. Sa supériorité provient de la manipulation partiellement simultanée des 2 lots d'animaux puis du simple équipement (un faisceau pour 2 stalles). En outre, la productivité élevée en moyenne (182 brebis/heure) demeure relativement constante (156 à 206 brebis/heure). Avec l'installation $12 \times 12$, la productivité, non seulement plus faible en moyenne (157 brebis/heure) devient aussi plus variable (127 à 195 brebis/heure). Compte tenu de nos résultats antérieurs (LE Du, 1984), on pourrait espérer l'accroître de 7 brebis/heure environ avec une race de type Manech. En utilisant 6 au lieu de 12 faisceaux, elle diminuerait légèrement pour des productions laitières (ou des temps d'émission du lait) comparables aux nôtres ( -7 brebis/heure), puis plus fortement si les productions sont très élevées car une attente deviendrait nécessaire entre chaque dépose.

D) Finalement, nous estimons qu'une installation constituée d'un seul quai de $4,5 \mathrm{~m}$ de long équipé d'un cornadis mobile à 12 places et de 12 faisceaux constitue une solution raisonnable pour traire un troupeau de taille moyenne. Avec 150 brebis, en estimant à une demi-heure le temps de mise en route et de nettoyage, le temps consacré à une traite serait toujours compris entre 1 h 15 min (à 195 brebis/heure) et $1 \mathrm{~h} 40 \mathrm{~min}$ (à 127 brebis/heure). Par comparaison avec la fosse la plus courante (24 stalles et 12 faisceaux-trayeurs), 3 constructeurs (3) chiffrent la diminution de l'investissement à 14 p. 100 en moyenne (installation complète avec lavage en circuit fermé, réception du lait dans une chambre de réception, sans tank à lait). La diminution de la surface du bâtiment (salle des machines comprise) de l'ordre de 27 p. 100 (43 $\mathrm{m}^{2}$ contre $59 \mathrm{~m}^{2}$ ) représente une économie qui n'a pas été chiffrée.

Certaines races, caractérisées par une grande capacité citernale, semblent très faciles à traire. VALLERAND (1984) cite comme exemple la brebis Corse qui donne son lait très rapidement. CASU et al. (1978) considèrent que la brebis Sarde peut se traire en supprimant toute forme d'égouttage. Dans de tels cas, on conçoit que les installations de traite traditionnelles conçues pour la Lacune s'implantent difficilement lorsqu'il s'agit de petits troupeaux. Notre travail mériterait par conséquent d'être poursuivi pour déterminer le nombre minimum de stalles et de faisceaux qui seraient compatibles avec une organisation rationnelle du travail.

Reçu en novembre 1985. Accepté en mars 1986.

\section{Summary}

Productivity of a milker in two small sized milking parlours for ewes

The hourly productivity of a milker was compared in two small sized milking parlours for ewes :

- $12 \times 24$ installation (fig. 1) : "Casse» system composed of two platforms with 12 milking stands and 6 teatcup clusters each (total : 12 clusters and 24 stands).

(3) Alfa-Laval, Z.I. du Chêne Sorcier, D.D. 161, 78340 Les Clayes-sous-Bois. Fullwood, 2, avenue Jean-Moulin, 94120 Fontenay-sous-Bois. Diabolo-Manus, 26, rue des Osiers, Coignières, 78130 Maurepas. 
$-12 \times 12$ installation (fig. 1) composed of a single platform with 12 stands and 12 clusters.

The results showed that :

- The productivity was higher (fig. 2) with the $12 \times 24 \quad(\mathrm{P}<0.001)$ than with the $12 \times 12$ installation (182 vs 157 ewes/hour). Exit times of ewes were shorter and the milker worked faster using one cluster for 2 stands $(12 \times 24)$.

- With 9 and $15 \mathrm{~h}$-intervals between milking, the productivity was higher in the evening than in the morning (177 vs 162 ewes/hour) $(\mathrm{P}<0.001)$. This seemed to be related to the consistency of the udder which was more engorged in the morning leading to a more difficult stripping and to more frequent cluster falls $(+43$ p. 100).

- The difference of productivity between milkers $(7.4 \mathrm{ewes} / \mathrm{hour})$ was rather due to the time spent for the admission $(P<0.05)$ and exit $(P<0.001)$ of the ewes than to the milking routine (N.S.). Productivity seemed to be much affected by the capacity of the milker to move easily from the pit the mobile cornadis, the gates and the concentrate feeder.

- With a simple method of milking, many breeds could be milked in cheaper parlours than those originally designed for the Lacaune breed. In the mediterranean area, an installation composed of 12 stands for 12 clusters appeared to be a good compromise for most herds of less than 150 ewes where mechanical milking tends to be introduced.

Key words : Ewe, milking installation, work organization, productivity.

\section{Références bibliographiques}

Anon, 1967. Système Casse. Traite mécanique des brebis. Ed. Société des Caves et Producteurs de Roquefort, $48 \mathrm{pp}$.

Bosc J., 1963. Problèmes de l'organisation du travail dans un troupeau de brebis laitières. Adaptation de la méthode "Arête de poisson》 à la traite mécanique des brebis. Compte rendu à l'A.F.Z., 3-39.

Bosc J., 1974. Organisation et productivité du travail de la traite des brebis laitières. Importance du choix d'une méthode de traite. Symp. Int. Traite Mécanique Petits Ruminants. Millau. Ann. Zootech., Numéro Hors Série, 231-251.

Casu S., Sanna A., Sanna L., Picinelli G., 1978. Simplification des opérations de la traite mécanique des brebis Sardes : la suppression de l'égouttage à la machine. Symp. Int. Traite Mécanique Petits Ruminants, Alghero, F.N.O.C.L., Paris, 215-223.

Cavalie J.L., Pascal R., 1978. Etude ergonomique de l'atelier de traite. Incidence sur l'homme de l'évolution technologique. Symp. Int. Traite Mécanique Petits Ruminants, F.N.O.C.L., Paris, 502-508.

Cottrer M., Delmas C., 1981. Le développement de la traite mécanique des brebis et

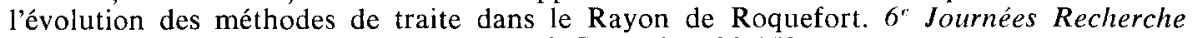
Ovine et Caprine, Ed. I.T.O.V.I.C.-S.P.E.O.C., Paris, 138-152.

Delmas C., 1984. Etude économique sur le développement de la mócanisation de la traite des brebis dans la zone de Roquefort. Symp. Int. Traite Mécanique Petits Ruminants, Valladolid, Espagne, Ed. Sever Cuesta, 228-250.

Delmas C., Poussou C., 1984. Une nouvelle installation de traite pour les petits troupeaux de brebis. Symp. Int. Traite Mécanique des Petits Ruminants, Valladolid, Espagne, Ed. Sever Cuesta, 326-332.

GAUTIER P., 1980. Etude de marché : salle de traite alternative de type Leducteur. Rapport de stage. INA Paris-Grignon, $47 \mathrm{p}$.

Labussiere J., 1984. Etudes des aptitudes laitières et de la facilité de traite de quelques races de brebis du «Bassin Méditerranéen ». Résultats préliminaires obtenus au 16 mai 1983. Symp. Int. Traite Mécanique Petits Ruminants, Valladolid, Espagne, Ed. Sever Cuesta, 730-794. 
LE Du J., 1977. Essais de manchons trayeurs pour brebis dans le Sud Aveyron. Rapport, INRA, Rennes, $17 \mathrm{pp}$.

Le Du J., Labussiere J., Douaire M., Combaud J.F., 1978. Effet de la conception de l'embouchure du manchon trayeur sur les caractéristiques de traite des brebis Préalpes du Sud. Ann. Zootech., 27, 571-581.

LE Du J., 1979. L'équipement de traite pour brebis : du nouveau. L'Elevage Bovin, 82, $85-93$.

LE Du J., 1981. Les salles de traite pour brebis. Journées Recherche Ovine et Caprine, Toulouse. Ed. I.T.O.V.I.C-S.P.E.O.C., Paris, 129-137.

LE Du J., 1982. Comparaison de matériels de traite pour brebis: manchons en silicone ou en caoutchouc, gobelets en inox ou en plastique. Ann. Zootech., 31, 139-148.

LE DU J., 1983. Comparaison d'un lactoduc en ligne haute et en ligne basse. Incidence sur la traite mécanique des brebis de races Lacaune et Manech. Ann. Zootech., 32, 43-52.

Le Du J., 1984. Etude de la productivité en salle de traite pour brebis : incidence du trayeur, de la race et de la taille de l'installation. Symp. Int. Traite Mécanique des Petits Ruminants, Valladolid, Espagne. Ed. Sever Cuesta, 303-314.

Mazac J., Kopal V., Cerny J., 1967. Rapport $\mathrm{n}^{\circ} 39$ sur les essais comparatifs internationaux des machines et des installations de traite pour brebis (en tchèque). Prague. Cité par MikUs M., 1974.

Mikus M., 1974. Machines à traire les brebis et les chèvres. Symp. Int. Traite Mécanique Petits Ruminants, Millau. Ann. Zootech., numéro hors série, 181-194.

Vallerand F., 1984. Les problèmes de mécanisation de la traite dans les systèmes laitiers extensifs. Symp. Int. Traite Mécanique Petits Ruminants, Valladolid, Espagne. Ed. Sever Cuesta, 216-227. 